\title{
Kaposi's sarcoma presenting with multiple cervical lymphadenopathies in a renal transplant recipient: a case report
}

\author{
TL Leung *, MRCP (UK), LY Wong, FHKAM (Medicine), A Cheuk, FHKAM (Medicine) \\ Department of Medicine and Geriatrics, Princess Margaret Hospital, Laichikok, Hong Kong \\ * Corresponding author: aaronleungt|@hotmail.com
}

Hong Kong Med J 2020;26:146-8

https://doi.org/10.12809/hkmj197971

\section{Case report}

A 57-year-old man underwent cadaveric renal transplantation in January 2018 and was prescribed mycophenolate mofetil, tacrolimus, and prednisolone as post-transplant immunosuppressive therapy. He developed multiple cervical lymphadenopathies at 6 months after transplantation. Fine needle aspiration cytology of the left submandibular lymph node, performed in the private sector, revealed only a hypocellular lesion. Considering the possibility of a post-transplantation lymphoproliferative disorder, excisional biopsy was arranged in our unit, but the patient defaulted from his appointment.

The patient attended the emergency department 9 months after transplantation complaining of shortness of breath. Physical examination on admission revealed generalised lymphadenopathy. Chest radiograph showed left lower, left middle, and right lower zone infiltrates. Despite the use of empirical piperacillin/tazobactam and withholding of mycophenolate mofetil, his condition deteriorated with worsening type one respiratory failure and increasing bilateral lung infiltrates on serial chest radiographs. Tacrolimus was discontinued. However, serial procalcitonin levels were undetectable. Computed tomography scan of the thorax suggested focal consolidative changes with diffuse cervical, axillary, mediastinal, hilar and abdominal lymphadenopathy (Fig 1). Biopsy of the groin and cervical lymph node showed spindle cell proliferation associated with surrounding vascular channels, red cell extravasation between spindle cells (Fig 2a), and positive human herpesvirus 8 (HHV-8) staining (Fig 2b). This confirmed the diagnosis of Kaposi's sarcoma (KS). There was no plasmablastic histopathology to suggest the presence of multicentric Castleman disease. Despite maximum supportive therapy with mechanical ventilation, empirical antimicrobials and antifungal treatment, his condition further deteriorated and he succumbed due to respiratory failure.

\section{Discussion}

Immunosuppressive therapy is known to increase the risk of infection and malignancy. In a study of

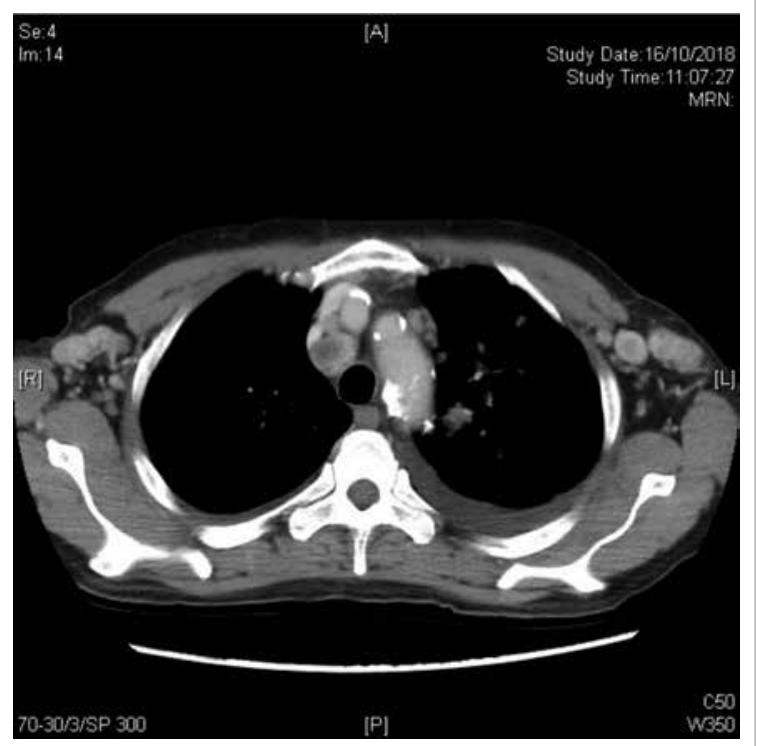

FIG I. Computed tomography image of thorax showing diffuse mediastinal lymphadenopathy

incidence of malignancy among a cohort of Hong Kong kidney transplant recipients from 1972 to 2011, the most prevalent malignancies were nonHodgkin's lymphoma followed by colorectal cancer, lung cancer, kidney cancer, and non-melanoma skin cancer. Only five cases of KS were reported. ${ }^{1}$ The low incidence of $\mathrm{KS}$ may be due to the low prevalence of HHV-8 seroprevalence in Asia. ${ }^{2}$ There were only 68 reports of KS in Hong Kong between 1983 and 2016 according to the Hong Kong Cancer Registry. In this report, we describe a case of $\mathrm{KS}$ in a renal transplant recipient who presented with multiple cervical lymphadenopathies.

In the clinical context of multiple cervical lymphadenopathies in a post-transplant recipient, post-transplant lymphoproliferative disorder, and multi-centric Castleman disease are our initial top differential diagnoses. However, the histology of the lymph node of our patient did not suggest these diagnoses but KS. The clinical presentation of post-transplant KS can be divided into exclusive 


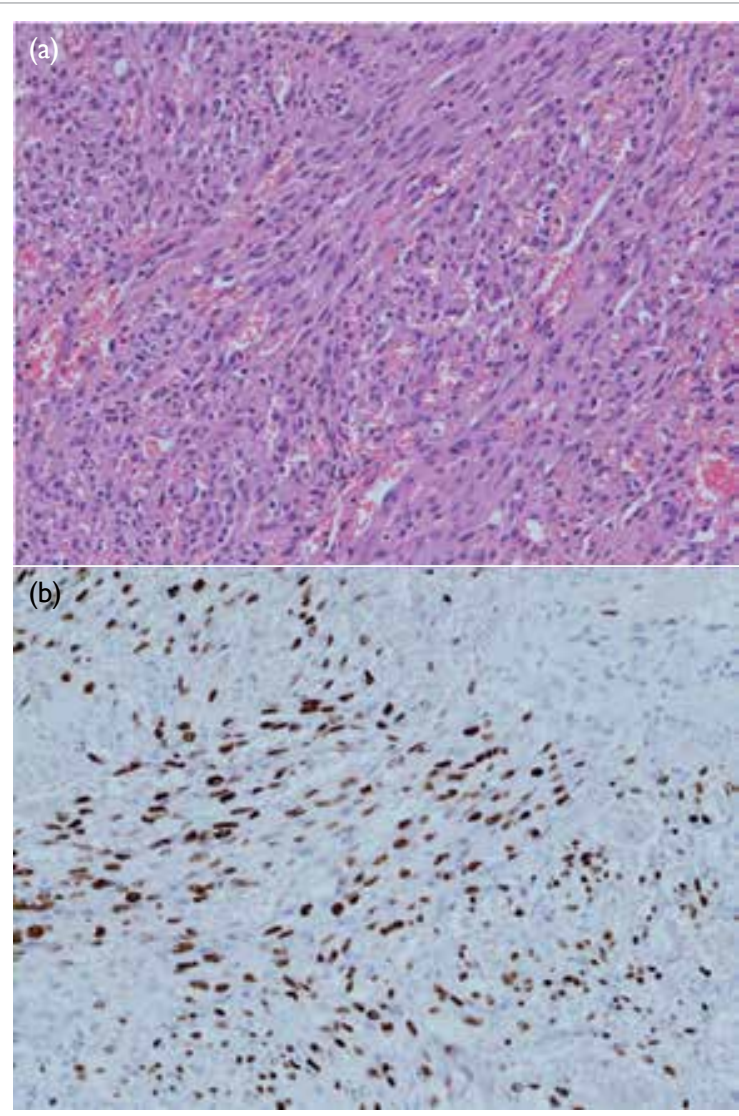

FIG 2. Photomicrographs showing (a) haematoxylin-eosin stained low-power view of fascicles of spindle cells with uniform nuclei forming slit-like vascular spaces and (b) diffuse immunohistochemical staining for human herpesvirus 8
12 to 39 months after transplantation. ${ }^{3,4,10}$ There is a male preponderance with a male-to-female ratio of 3:1. ${ }^{4}$ The 5 -year survival rate is around $70 \%$, although those with visceral involvement generally carry a poorer prognosis. ${ }^{12}$

There are no established guidelines for treatment of post-transplant KS so treatment often depends on clinical presentation. Tapering or withdrawal of immunosuppressants is the mainstay of therapy. Intralesional chemotherapy may be used for a single dermatological lesion. Systemic chemotherapy, such as liposomal anthracycline or taxanes, may be considered for widespread disease. Complete remission with immunosuppressant reduction or withdrawal has been reported as $50 \%$ to $60 \%$ and graft loss as $20 \%$ to $30 \%$ in the premammalian target of rapamycin (mTOR) inhibitor era.,10 Treatment with mTOR inhibitor, such as sirolimus, has gained recognition with its antiangiogenic and anti-neoplastic activity, particularly among patients with an exclusive dermatological presentation. Three previous case series of 25 patients reported a $100 \%$ remission rate after switching from a calcineurin inhibitor to sirolimus, while two patients had graft loss due to causes other than rejection. ${ }^{3,5,6}$ Other case series have reported treatment failure with sirolimus. Visceral involvement and delay in switching from calcineurin inhibitor to mTOR inhibitor after diagnosis of KS may contribute to treatment failure. ${ }^{9}$ Further study is essential to determine the optimal treatment for post-transplant KS, especially for those with visceral involvement.

\section{Conclusion}

Post-transplant KS presenting with multiple cervical lymphadenopathies is rare and may signify an aggressive subtype. Withdrawing immunosuppressive therapy alone failed to salvage our patient. Further study is required to evaluate the potential of switching mycophenolate mofetil and calcineurin inhibitor to mTOR inhibitor to improve the prognosis for this subgroup of patients.

\section{Author contributions}

All authors contributed to the concept of the study, acquisition and analysis of the data, drafting of the manuscript, and critical revision of the manuscript for important intellectual content. All authors had full access to the data, contributed to the study, approved the final version for publication, and take responsibility for its accuracy and integrity.

\section{Acknowledgement}

We thank Dr Matthew KL Tong, Dr Hilda WH Chan, Dr Hon-lok Tang, and Dr Samuel KS Fung for their valuable opinions and supervision on writing the manuscript. We thank Dr Yuen-fun Mak for her expert guidance on pathology review. 


\section{Declaration}

Part of the content about this case was presented in the Nephrology interhospital meeting in September 2019.

\section{Conflict of interest}

All authors have disclosed no conflicts of interest.

\section{Funding/support}

This case report received no specific grant from any funding agency in the public, commercial, or not-for-profit sectors.

\section{Ethical approval}

The patient was treated in accordance with the Declaration of Helsinki. The patient provided informed consent for all procedures.

\section{References}

1. Cheung CY, Lam MF, Chu KH, et al. Malignancies after kidney transplantation: Hong Kong renal registry. Am J Transplant 2012;12:3039-46.

2. Zhang $\mathrm{T}$, Shao $\mathrm{X}$, Chen $\mathrm{Y}$, et al. Human herpesvirus 8 seroprevalence, China. Emerg Infect Dis 2012;18:150-2.

3. Stallone G, Schena A, Infante B, et al. Sirolimus for Kaposi's sarcoma in renal-transplant recipients. N Engl J Med 2005;352:1317-23.

4. Penn I. Sarcomas in organ allograft recipients. Transplantation 1995;60:1485-91.
5. Yaich S, Charfeddine K, Zaghdane S, et al. Sirolimus for the treatment of Kaposi Sarcoma after renal transplantation: a series of 10 cases. Transplant Proc 2012;44:2824-6.

6. Gutiérrez-Dalmau A, Sánchez-Fructuoso A, SanzGuajardo A, et al. Efficacy of conversion to sirolimus in posttransplantation Kaposi's sarcoma. Transplant Proc 2005;37:3836-8.

7. Barete $\mathrm{S}$, Calvez V, Mouquet $\mathrm{C}$, et al. Clinical features and contribution of virological findings to the management of Kaposi sarcoma in organ-allograft recipients. Arch Dermatol 2000;136:1452-8.

8. Stebbing J, Mazhar D, Lewis R, et al. The presentation and survival of patients with non-cutaneous AIDS-associated Kaposi's sarcoma. Ann Oncol 2006;17:503-6.

9. Lebbé C, Euvrard S, Barrou B, et al. Sirolimus conversion for patients with posttransplant Kaposi's sarcoma. Am J Transplant 2006;6:2164-8.

10. Montagnino G, Bencini PL, Tarantino A, Caputo R, Ponticelli C. Clinical features and course of Kaposi's sarcoma in kidney transplant patients: report of 13 cases. Am J Nephrol 1994;14:121-6.

11. Qunibi W, Akhtar M, Sheth K, et al. Kaposi's sarcoma: the most common tumor after renal transplantation in Saudi Arabia. Am J Med 1988;84:225-32.

12. Woodle ES, Hanaway M, Buell J, et al. Kaposi sarcoma: an analysis of the US and international experiences from the Israel Penn International Transplant Tumor Registry. Transplant Proc 2001;33:3660-1. 